\title{
Aneurysmal bone cyst arising in iliopubic chondromyxoid fibroma - a case report
}

\author{
João Soares do Vale ${ }^{\mathrm{a},{ }^{*}}$, Sara Diniz ${ }^{\mathrm{a}}$, Filipe Rodrigues ${ }^{\mathrm{a}}$, Ana Ribau ${ }^{\mathrm{a}}$, André Coelho ${ }^{\mathrm{b}}$, Vânia Oliveira ${ }^{\mathrm{a}}$, \\ Pedro Cardoso ${ }^{\text {a }}$
}

${ }^{a}$ Department of Orthopaedics, Porto Hospital and University Center, Porto, Portugal.

${ }^{b}$ Department of Pathologic anatomy, Porto Hospital and University Center, Porto, Portugal.

\begin{abstract}
Chondromyxoid fibroma is rare cartilaginous tumor, accounting for $0.5 \%$ of all primary bone tumors and $2 \%$ of benign bone tumors. Areas of aneurysmal bone cysts (ABC) may be found within CMF in $8.6 \%$ of cases. A 20 -year-old man presents pain due to a mass on iliopubic ramus that was diagnosed as an aneurysmal bone cyst arising from a chondromyxoid fibroma. This case confirms the rare association between aneurysmal bone cyst and chondromyxoid fibroma. Although aneurysmal bone cyst is more frequently associated with highly vascularized tumors, it is important to consider the diagnosis in the presence of chondromyxoid fibroma.

Keywords: Chondromyxoid fibroma; scecondary aneurysmal bone cyst; iliopubic ramus
\end{abstract}

\section{INTRODUCTION}

Chondromyxoid fibroma (CMF) was described by Jaffe and Lichtenstein as a distinctive entity in $1948^{[1]}$. The definition given by the World Health Organization (WHO) is a benign cartilaginous neoplasm, composed of lobules formed by spindle-shaped cells with myofibroblastic features at the periphery, and stellate and chondrocyte-like cells towards the center, the extracellular matrix of the lobules are fibrous at the periphery and myxoid and chondroid towards the center ${ }^{[2]}$.

This rare cartilaginous tumor, accounting for $0.5 \%$ of all primary bone tumors and $2 \%$ of benign bone tumors, occurs predominantly in adolescents and young adults and more commonly in males ${ }^{[2,3]}$. This tumor can occur at almost any osseous site, with a predilection for bones of the lower extremities, usually the proximal tibia ${ }^{[2,3]}$. Flat-bones account for 25 to $30.3 \%$

\footnotetext{
* Corresponding author: João Soares do Vale Mailing address: Rua Padre Cruz, 148 1o, 4050-219 Porto, Portugal.

E-mail: jsoaresvale@gmail.com

Received: 15 April 2021 / Accepted: 26 May 2021
}

of cases, with ilium been the most affected ${ }^{[2,4,5]}$. Areas of aneurysmal bone cysts (ABC) may be found within CMF in $8.6 \%$ of cases ${ }^{[4]}$.

$\mathrm{ABCs}$ is the result of specific pathologic change, which is probably the result of trauma or a tumor-induced anomalous vascular process ${ }^{[6]}$. The most common is giant cell tumor, but others like osteoblastoma, angioma, chondroblastoma, telangiectatic osteosarcoma are common too ${ }^{[6]}$.

To the best of our knowledge, there is not any case of an aneurysmal bone cyst arising from an iliopubic chondromyxoid fibroma.

\section{CASE REPORT}

A 20-year-old man with no relevant medical history presents mechanical pain in the left inguinal region with 6 months of evolution. Physical examination revealed a $4 \mathrm{~cm} \times 2 \mathrm{~cm} \times 2 \mathrm{~cm}$ palpable mass in the groin. The conventional radiography (Figure 1) revealed an osteolytic tumor in the left ilio-pubic ramus, with cortical thinning and a fracture line in the central region. MRI presents a well-defined lesion with lobulated contour and internal septation (Figure 2) suggesting an aneurysmal bone cyst or telangiectatic osteosarcoma. Ultrasonography-guided percutaneous biopsy diag- 


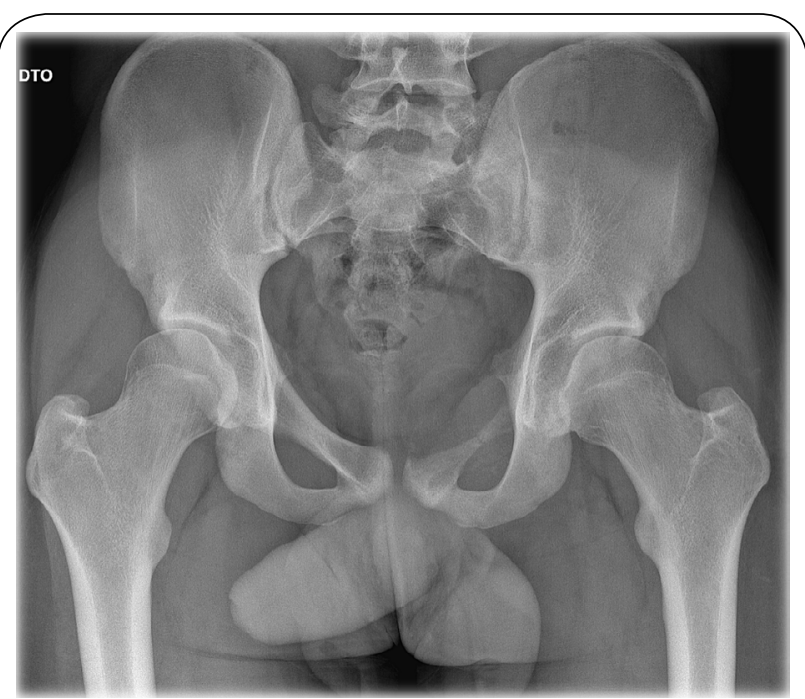

Figure 1. Preoperative radiographic image shows thinning of the iliopubic ramus and a fracture line in the central region.

nosed a chondromyxoid fibroma.

The tumor excision was performed through an anterior approach. A Pfannenstiel incision was extended to the proximal region of the left thigh, in order to isolate and protect the femoral neurovascular bundle. The spermatic cord was recognized and protected. The bladder was protected as well. Pubic symphysis and the anterior rim of the acetabulum were identified and used as anatomical references to osteotomies. An enbloc resection of iliopubic ramus and the soft tissues that surrounded it was made (Figure 3). No pelvic ring reconstruction was considered for mechanical purposes. The abdominal wall was reinforced with Gore$\mathrm{Tex}^{\circledR}$ mesh, that was attached to symphysis, anterior acetabular rim and abdominal wall muscle fascia to prevent intra-pelvic herniation.

The patient was discharged home 2 days after surgery, with partial weight bearing and no evidence of complications.
Microscopic examination showed complete excision of the lesion, with free margins.

Histological analysis revealed a benign mesenchymal neoplasm, well delimitated and organized in chondromyxoid lobes of variable cellularity, with stellate cells in myxoid areas and lacunar cells in chondroid areas features consistent with chondromyxoid fibroma (Figure 4A and 4B). There were also abundant hemorrhagic cavitated areas developing within the tumor, with septa rich in osteoclastic-type multinucleated giant cells and with deposition of linear immature osteoid, typical of aneurysmal bone cyst transformation (Figure 4C and 4D), explaining the images seen in MRI. The neoplasm occupied and expanded the ischio-pubic ramus, reaching the subcartilaginous area of the pubic symphysis, but it didn't invade the adjacent soft tissue, as it was surrounded by a thin layer of woven bone, a consequence of cortical bone remodeling.

After 1 year of follow-up, the patient is pain-free, without functional impairment or complications. There is no recurrence until the present.

\section{DISCUSSION}

This case represents an unusual localization for this type of tumor. A literature review of 278 cases of CMF of bone, reported that $30.3 \%$ of cases occurred in flat bones. Of these only 1 case $(1.2 \%)$ was located on the pubis and 2 on ischium $(2.4 \%){ }^{[4]}$.

As the name indicates, chondromyxoid fibromas show a variety of histological features. The classic histological feature is stellate or spindle-shaped cells arranged in lobules in a myxoid background ${ }^{[4]}$. However, a lobulated pattern was seen in only $86.7 \%$ of cases, and can present a macro or microlobular pattern isolated or a mixture ${ }^{[4]}$.

In our case, the tumor was accompanied by an area of $\mathrm{ABC}$. The origin of the term $\mathrm{ABC}$ comes from an article

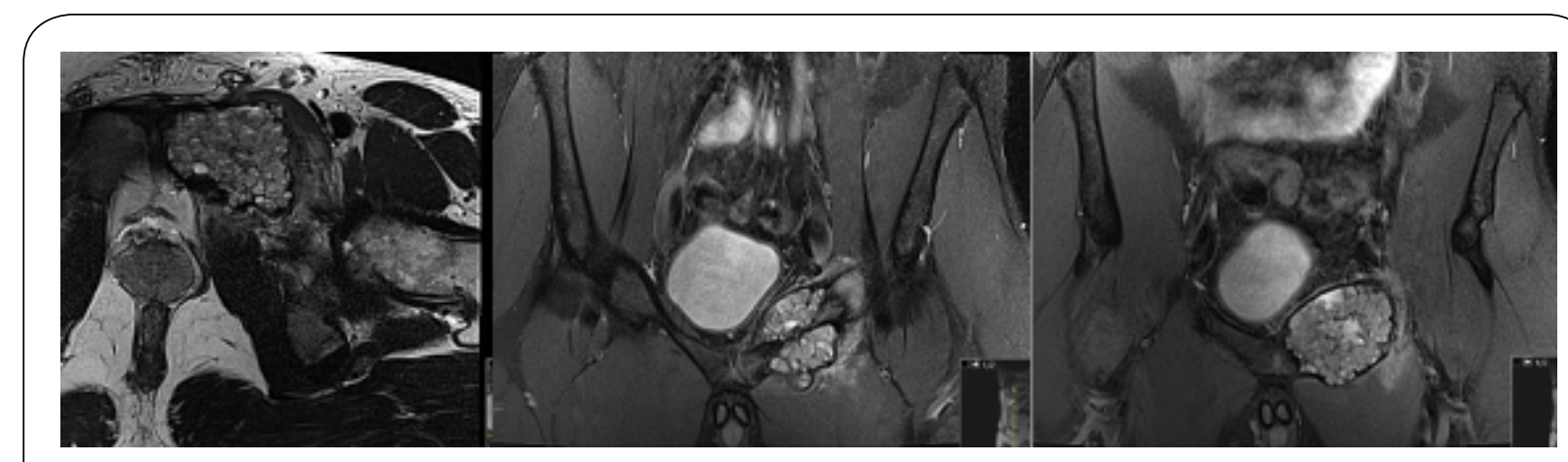

Figure 2. Preoperative MRI: weel defined lesion with lobulated contour and internal septation. 


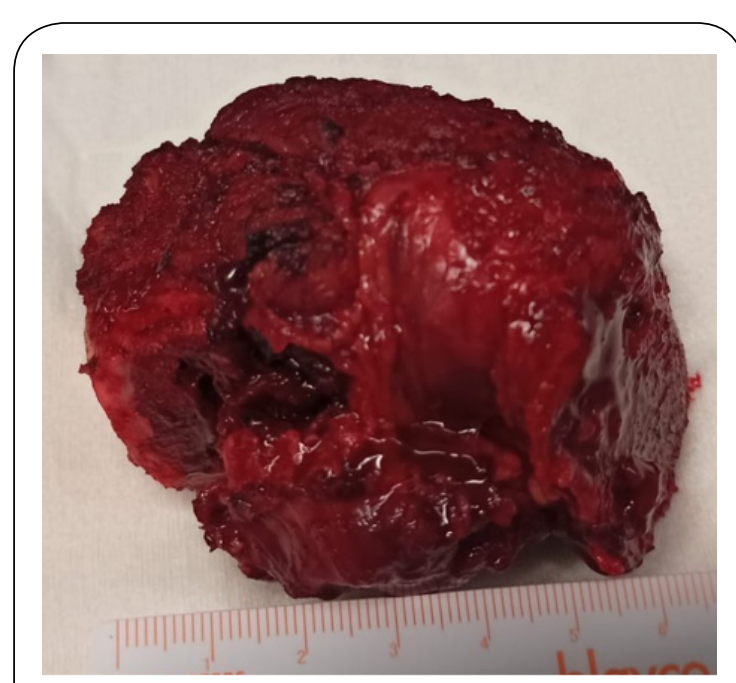

Figure 3. En-bloc resection of iliopubic ramus and the soft tissues that surrounded it. by Jaffe and Lichtenstein in $19422^{[7]}$. They postulated that $\mathrm{ABC}$ might be a secondary phenomenon due to hemorrhagic "blow-out" in a preexisting lesion, which may be destroyed in the process ${ }^{[7]}$. A preexisting lesion was identified in approximately one third ${ }^{[6]}$.

ABCs conventional treatment has been the surgical removal of the entire lesion or as much as possible, with recurrence seen in $10-70 \%$ of cases ${ }^{[2,6]}$. Spontaneous regression following incomplete removal is very unusual ${ }^{[2]}$. More recently, non-surgical polidocanol sclerotherapy demonstrates to be a safe and effective option even for aggressive ABCs, depending on location and surgical risks ${ }^{[8]}$. Appropriate treatment requires realizing that it has a specific pathophysiologic origin and identifying the preexisting lesion. If no coexisting lesion is identified, lesions are usually treated

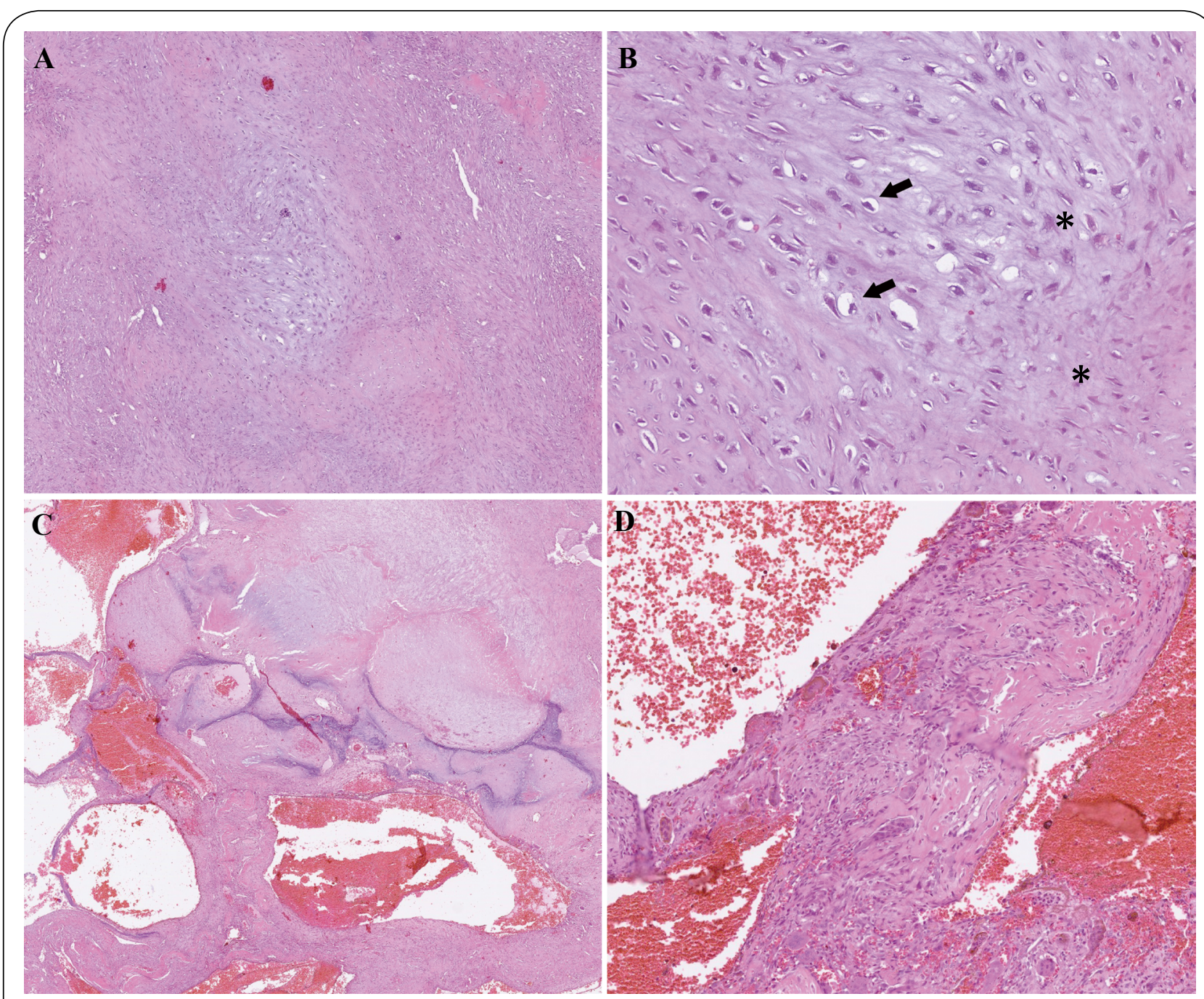

Figure 4. (A) The tumor has a zonal, vaguely lobulated architecture, with hypocellular chondromyxoid matrix at the center and a more cellular spindled-cell component at the periphery; (B) The chondroid central areas have oval to stellate cells $(*)$, some of them residing within lacunae (arrow) surrounded by cartilage-like matrix; (C) Secondary aneurysmal bone cyst-like areas are seen throughout the neoplasm, with hemorrhagic spaces; (D) In these areas, the septa contain numerous osteoclastic-type multinucleated giant cells, abundant hemosiderin and linear deposition of osteoid matrix. 


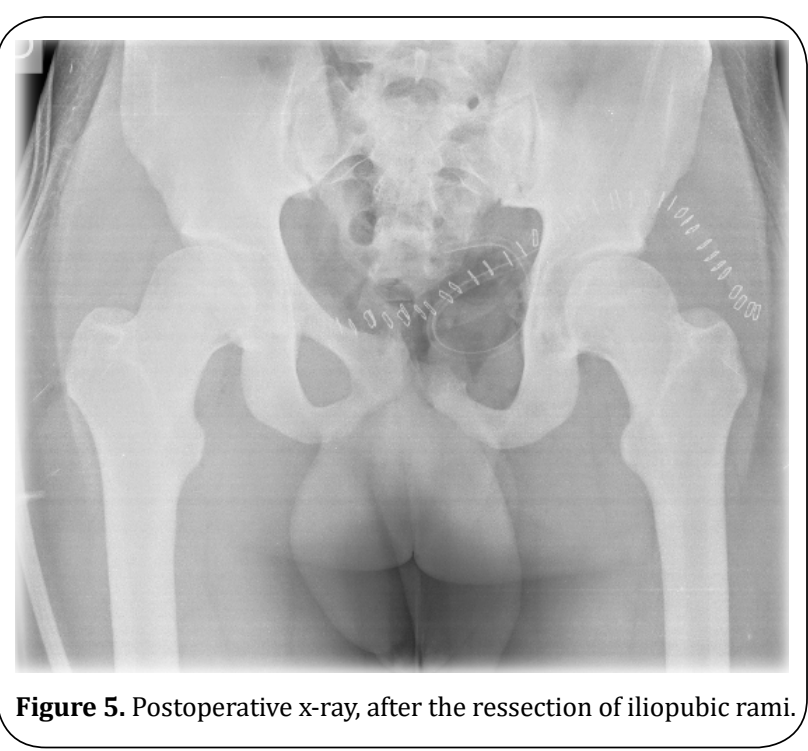

with curettage and bone grafting ${ }^{[6]}$.

The typical treatment of CMF by curettage has a $20 \%$ to $25 \%$ recurrence rate, lowering to $7 \%$ with the addition of bone grafting ${ }^{[9,10]}$. Resection provides lower recurrence rates but is not always feasible.

Based on the system proposed by Enneking WF and Dunham WK, this case of CMF involves zone III (involvement of pubis and ischium bones) ${ }^{[11]}$. A review of 8 cases describing surgical management of CMF of the pelvis, reported two cases of involvement of superior ramus of the pubis. One was treated with resection without grafting and complicated with pelvic internal organs herniation. In the second case, reconstruction was done using fibular strut allograft, with no complication on follow-up ${ }^{[12]}$. Another case of CMF of iliopubic ramus presented good results with aggressive curettage and chip bone grafting. The author reclaims this procedure as safe, easy, and less morbid for this anatomic site ${ }^{[13]}$.

In our case, we resected iliopubic ramus and used a Gore-tex ${ }^{\circledast}$ surgical mesh to prevent intra-pelvic herniation. Resection should be performed for large lesions or in anatomic sites such as the complex pelvis. Such a procedure does not increase the risk of fracture and reduces the likelihood of recurrence or a second surgery.

The secondary $\mathrm{ABC}$ arising from $\mathrm{CMF}$ was verified histologically. It is associated with a significant recurrence rate and the surgical strategy adopted was then confirmed the most appropriate.

This case represents a rare tumor, in an uncommon location, with an atypical histological transformation. This case shows the rare association between $\mathrm{ABC}$ and
CMF. Although secondary ABCs are more frequently associated with highly vascularized tumors, it is important to consider the diagnosis of CMF in the presence of an $A B C$, as it may alter the clinical approach to minimize the risk of recurrence.

\section{DECLARATIONS}

\section{Authors' contributions}

João Vale: Wrote the manuscript, Performed the literature review;

Sara Diniz: Edited the paper;

Filipe Rodrigues: Edited the manuscript;

Ana Ribau: Edited the paper;

André Coelho: Edited the manuscript;

Vânia Oliveira: Edited the manuscript;

Pedro Cardoso: Edited the manuscript.

\section{Conflict of interest}

All authors declared that there are no conflicts of interest.

\section{Ethics approval}

Written informed consent was obtained from the patient for publication of this case report and any accompanying images.

\section{REFERENCES}

1. HL, J. (1948). Chondromyxoid fibroma of bone. A distinctive benign tumor likely to be mistaken especially for chondrosarcoma. Arch Pathol, 45, 541-551.

2. Spieler, P., \& Rössle, M. (2012). Soft Tissue and Bone. In Nongynecologic Cytopathology (pp. 1053-1105). Springer, Berlin, Heidelberg.

3. Greenspan, A., Jundt, G., \& Remagen, W. (2007). Differential diagnosis in orthopaedic oncology. Lippincott Williams \& Wilkins.

4. Wu, C. T., Inwards, C. Y., O’laughlin, S., Rock, M. G., Beabout, J. W., \& Unni, K. K. (1998). Chondromyxoid fibroma of bone: a clinicopathologic review of 278 cases. Human pathology, 29(5), 438-446.

5. Zillmer, D. A., \& Dorfman, H. D. (1989). Chondromyxoid fibroma of bone: thirty-six cases with clinicopathologic correlation. Human pathology, 20(10), 952-964.

6. Kransdorf, M. J., \& Sweet, D. E. (1995). Aneurysmal bone cyst: concept, controversy, clinical presentation, and imaging. AJR. American journal of roentgenology, 164(3), 573-580.

7. JAFFE, H. L., \& Lichtenstein, L. (1942). Solitary unicameral bone cyst: with emphasis on the roentgen picture, the pathologic appearance and the pathogenesis. Archives of Surgery, 44(6), 1004-1025. 
8. Brosjö, O., \& Tsagozis, P. (2014). Treatment of an aggressive aneurysmal bone cyst with percutaneous injection of polidocanol: a case report. Journal of medical case reports, 8(1), 1-4.

9. Subach, B. R., Copay, A. G., Martin, M. M., Schuler, T. C., \& Romero-Gutierrez, M. (2010). An unusual occurrence of chondromyxoid fibroma with secondary aneurysmal bone cyst in the cervical spine. The Spine Journal, 10(2), e5-e9.

10. Gherlinzoni, F., Rock, M., \& Picci, P. (1983). Chondromyxoid fibroma. The experience at the Istituto Ortopedico Rizzoli. JBJS, 65(2), 198-204.
11. Enneking, W. F., \& Dunham, W. K. (1978). Resection and reconstruction for primary neoplasms involving the innominate bone. The Journal of bone and joint surgery. American volume, 60(6), 731-746.

12. Jamshidi, K., NAJD, M. F., \& JAFARI, D. (2015). Chondromyxoid fibroma of pelvis, surgical management of 8 cases. Arch Iran Med, 18(6), 367-370.

13. Arikan, M., Toğral, G., Yildirim, A., \& Aktaş, E. (2016). Chondromyxoid fibroma of the pubic ramus: a case report and literature review. Acta Orthop Traumatol Turc, 50(1), 115-119. 